\title{
OSTEOPOROSIS: UNA PATOLOGÍA CON AFECTACIÓN PERSONAL, FAMILIAR, LABORAL Y SOCIAL
}

\author{
MIRA FERRER, LUZ Ma . $\left.^{*}\right)$ DE LA FUENTE DÍEZ, ELENA.(**)
}

(*) Médico Adjunto Especialista en Obstetricia y Ginecología. Sección de menopausia y patología endometrial.

Servicio de Obstetricia y Ginecología. Consorcio Hospital General Universitario de Valencia.

(**) Médico Interno Residente en Obstetricia y Ginecología. Servicio de Obstetricia y Ginecología.

Consorcio Hospital General Universitario de Valencia..

\section{RESUMEN}

La osteoporsis y las fracturas osteoporóticas son un importante problema en nuestra sociedad debido a su gran frecuencia. En nuestro siglo la población europea y española está envejeciendo y la prevalencia de la osteoporosis va en aumento. Por otro lado la osteoporosis afecta a la población con edad a partir de la década de los 40 , es decir durante la vida laboral activa, así que representa una importante causa de absentismo laboral. Esto produce un alto coste económico, social, familiar y sanitario. Es interesante promover programas de detección para disminuir estas consecuencias. Un programa efectivo de diagnóstico y tratamiento de la osteoporosis es el método "gold tandard" para el manejo de esta "enfermedad silenciosa". La detección precoz de los factores de riesgo de esta enfermedad es el punto clave de actuación. Debemos recomendar hábitos de vida saludables para evitar la aparición de la osteoporosis y de las fracturas osteoporóticas. Los Servicios de Prevención de Riesgos Laborales tienen una posición privilegiada para instaurar actividades en este sentido.

\section{PALABRAS CLAVE:}

Osteporosis, fracturas osteoporóticas, osteopenia, factores de riesgo, prevención.

\section{SUMMARY}

The osteoporosis and the osteoporotic fracture represent a prevalent disease and one of the main problem in the developed countries. The European and Spanish population are going to be older from the last century and the osteoporosis is growing up. In other hand, usually, the disease affect people at the 40 th decade of life when they are working population in active labour life period, so the osteoporosis is an important cause of absenteeism, and consistently, the osteoporosis means a high economic, social, family, sanitary and labour cost. One of the main effectives activities in the control of the disease are the instauration of prevention programs guided to target populations. An effective program of early diagnosis and treatment of osteoporosis is the gold standard method to attend correctly this "silent illness". The roads points of actuation are related with an early detection of the risks factor in each patient before the fracture and others complications takes place. Them, we must recommend healthful habits of life to avoid the appearance of osteoporosis and osteoporosis fracture. The Services of Prevention of Labour Risk have a privileged position to develop this prevention programes.

\section{KEY WORDS:}

Osteoposis, osteoporotic fracture, osteopenia, risk factor, prevention. 


\section{ASPECTOS SOCIO-SANITARIOS}

La osteoporosis constituye un problema de salud de gran magnitud, dadas sus graves consecuencias sanitarias, económicas y asistenciales. Durante los últimos treinta años asistimos a un incremento mantenido de la población de riesgo, debido al incremento de la esperanza de vida de la población mayor de 50 años. Las mejoras en la prevención y en la asistencia sanitaria conllevan un aumento en las expectativas de vida y por lo tanto un envejecimiento de la población, derivando todo en un mayor requerimiento de atención socio-sanitaria, y un incremento del consumo de recursos económicos.

La incidencia de fracturas osteoporóticas se ha visto incrementada notablemente durante las últimas décadas, incremento que también afecta a poblaciones relativamente jóvenes que se encuentran profesionalmente activas, lo que despierta cierta inquietud si consideramos las repercusiones laborales y económicas, tanto desde la perspectiva social, económica, familiar y personal.

La perspectiva de la enfermedad desde el punto de vista sanitario es obvia, pero si analizamos sus repercusiones sociales y laborales desde su vertiente económica determinaremos su importancia actual.

Tan solo citar algunos ejemplos que nos ayuden a centrar el aspecto socio-económico de la enfermedad.

Se calcula un coste estimado del 8,8 \% del PIB, pero en los próximos años se estima que la osteoporosis y sus consecuencias (transporte, ayudas sociales, rehabilitación, etc.) podrán incrementar el 20\% de los gastos sufragados por la Sanidad Pública; lo que supondrá aproximadamente 40 millones de euros adicionales. Estos estudios se refieren, fundamentalmente, a los costes derivados del tratamiento de las fracturas de cadera, pero hay otros procesos clínicos relacionados con la enfermedad que presentan una elevada incidencia. Y aunque sus costes directos no sean tan importantes, el gasto generado por el gran número de casos, al final derivan en un coste muy importante.

En España, en 1995, el coste derivado de las fracturas osteoporóticas se estimó en 18 millones de euros, y en la actualidad se sabe que es superior a los 25 millones.

El coste derivado de las fracturas de extremidad distal de radio permanece más estable debido a que la mayoría de ellas se trata de forma ortopédica ambulatoriamente. Sin embargo, su alta incidencia, que alcanza el 6.6/1.000, supone un problema importante desde el punto de vista sanitario.

Según datos del Ministerio de Sanidad y Consumo, durante el periodo 1966-2000, el consumo de fármacos para el tratamiento de la osteoporosis se multiplicó por 16 , pasando de 0,14 dosis por 100.000 habitantes/día a 4,91 dosis por 100.000 habitantes/día, lo que supone, según los datos aportados por Fármaco-Economía (Osteoporosis y evaluación económica, diciembre del 2005), un cose total de 156,26 millones de euros para el año 2000 .

Esta situación, sin olvidar las razones sanitarias, ha propiciado la puesta en marcha de programas de prevención primaria y secundaria de fracturas osteoporóticas, lo que en parte justifica el incremento del gasto relativo.

En el futuro, estos planes de prevención supondrán una importante mejora sanitaria, social y laboral, así como un ahorro no desdeñable, al permitir disminuir las inversiones en atención terciaria orientadas, en su mayor parte, a paliar los efectos de secuelas, tratamientos de rehabilitación, cirugía reparadora, utilización de prótesis, problemas laborales y ayudas sociales.

\section{ASPECTOS CLÍNICOS}

A la hora de entender y centrar la enfermedad desde el punto de vista clínico, es importante tener en cuenta una serie de consideraciones.

La primera se centraría en saber cuando podemos considerar una perdida de masa ósea como osteoporosis, y atendiendo a los criterios establecidos por el Nacional Institute of Health (Conensus Conference 2001), la osteoporosis se define como un trastorno del esqueleto caracterizado por una alteración de la resistencia ósea, que predispone a una persona a un mayor riesgo de fracturas.

Otro concepto importante es la resistencia del hueso, que refleja principalmente la integración de la densidad y calidad ósea. La osteoporosis es la enfermedad ósea metabólica más frecuente.

El principal factor de riesgo de la osteoporosis es la osteopenia, que se define como baja masa ósea sin llegar a aumentar el riesgo de fractura. 
La definición de osteoporosis y osteopenia se basa en la medición de la densidad mineral ósea por densitometría (tabla 1).

\section{Tabla 1.}

Normal $\longrightarrow$ Escala $\mathrm{T}>-1$.
Osteopenia $\longrightarrow$ Escala $\mathrm{T}<-1 \mathrm{y}>-2,5$.
Osteoporois $\longrightarrow$ Escala $\mathrm{T}<-2,5$.
Osteoporosis grave $\longrightarrow$
$\begin{aligned} & \text { Escala } \mathrm{T}<-2,5 \mathrm{y} \\ & \text { fractura por fragilidad. }\end{aligned}$

La densidad mineral ósea ( DMO ) a cualquier edad, es el resultado de la combinación de dos variables, la masa ósea conseguida durante el crecimiento y el ritmo de pérdida posterior.

La resistencia ósea refleja la integración de dos elementos;

\section{- Densidad y calidad ósea.}

A su vez la calidad ósea engloba diversas características del tejido óseo relacionadas con la macroarquitectura (tamaño del cuerpo vertebral, longitud del eje femoral...), la microarquitectura (porosidad cortical, conectividad trabecular, etc.), el recambio óseo ( grado de resorción y de formación) y propiedades tisulares como por ejemplo el grado de mineralización ósea secundaria, el acúmulo de microlesiones y la distribución de los enlaces cruzados del colágeno.

Las consecuencias clínicas de la disminución de la densidad mineral ósea conducen a un aumento del riesgo de sufrir fracturas.

Las principales fracturas asociadas a osteoporosis son: fractura vertebral, fractura de fémur, fractura de Colles, fractura de húmero proximal y fractura costal.
La fractura vertebral tiene una elevada prevalencia , alrededor del $5 \%$ de ellas se presentan en la población en edad laboral. La mayoría de ellas son asintomáticas, y se desconoce la proporción exacta en que causan invalidez.

Además estas fracturas pueden ocasionar distintos problemas como pérdida de talla, magnificación de la cifosis torácica, de la lordosis cervical y lumbar, con sobrecarga del músculo romboides al desplazarse la escápula. También puede ocasionar disminución del volumen pulmonar total.

Finalmente, todo lo anterior puede derivar en pérdida de movilidad, discapacidad, dolor agudo y crónico.

La fractura de fémur es la consecuencia más grave de la osteoporosis por su gran morbi-mortalidad, y por los elevados costes económicos generados. Esto la convierte en el principal problema socio-sanitario relacionado con la enfermedad.

La fractura de Colles suele ser la primera en la cronología de las fracturas asociadas a osteoporosis. $\mathrm{La}$ fractura de húmero proximal y costal son menos frecuentes y de menor magnitud.

\section{FACTORES DE RIESGO}

Evitar las fracturas y sus consecuencias se ha convertido en el objetivo primordial.

Evitar las fracturas y sus consecuencias se ha convertido en el objetivo al que todos aspiramos, pero diagnosticar la ostoporosis antes de que aparezca la fractura es muy difícil, de ahí su nombre de "epidemia silenciosa".

Los factores de riesgo pueden ser de dos tipos: inherentes al hueso y factores extra-esqueléticos.

Los inherentes al hueso son: masa ósea, macroarquitectura, microarquitectura, recambio óseo y microfracturas, que afectan tanto a la cantidad como a la calidad del hueso.

De los factores extra-esqueléticos, algunos no son modificables (genética), otros actúan a lo largo de toda la vida (genética-dieta-ejercicio) y otros pueden afectar a partir de un momento determinado (fármacos, dieta, enfermedades). 
Tabla 2: Factores de riesgo.

Raza blanca.

Mujer.

Edad.

Delgadez, estatura baja, piel blanca.

Sedentarismo, inmobilidad.

Dieta baja en calcio.

Antecedentes familiares de osteoporosis y fracturas por esta causa.

Déficit estrogénico: menopausia precoz, anorexia, hiperprolactinemia.

Hábitos tóxicos: cafeína, alcohol, tabaco.

Enfermedades: Cushing, hiperparatiroidismo, hipertiroidismo, hipogonadismo, diabetes mellitus.

Pico de masa ósea inferior al correspondiente por la edad.

Fármacos: corticoides (incluidos los inhalatorios por tiempo prolongado ), heparina, antiepilépticos.

Genética.- Es de los factores de riesgo más importantes. Puede explicar hasta diferencias de más de un $50 \%$ en la consecución DMO.Sexo.- La osteoporosis es más prevalente en mujeres que en hombres, pero los varones tienen un mayor tamaño óseo y la osteporosis se relaciona con el mayor volumen de la masa ósea (Consensus conference 2001).

Edad.- El pico máximo de masa ósea se alcanza entre los 25-30 años, permanece estable hasta los 40, y a partir de aquí disminuye lentamente (aproximadamente, un $0,5 \%$ anual). Desde los 50 años se puede producir una pérdida de hasta un 3-5\%.

Raza.- La raza blanca y asiática son las que más prevalencia de osteoporosis tienen.
Menopausia.- Cuando disminuyen fisiológicamente los estrógenos en la mujer se produce una falta de su acción sobre los receptores de la célula ósea, alterando el "turnover óseo".

Hipoestronismo.- Cuando aparece un déficit estrogénico antes de producirse el pico máximo de masa ósea, este disminuye, produciéndose una alteración en su mantenimiento.

Delgadez..- Aumenta el riesgo de osteoporosis y fractura.

Tóxicos.- El tabaco se asocia con hipoestronismo y produce disminución de masa ósea. La cafeína y la teína también aumentan la excreción renal de calcio y por lo tanto disminuyen la recuperación de calcio por el hueso. El alcohol disminuye la formación ósea por hiperaldosteronismo, acidosis y alteraciones en el metabolismo de la vitamina D.

Fármacos.- Algunas fármacos aumentan el riesgo de osteoporosis y osteopenia por su efecto a nivel del túbulo renal, con lo que disminuye la formación y aumentan la resorción ósea.

Nutrición.- La ingesta inadecuada de calcio y vitamina $\mathrm{D}$ produce una disminución del aporte $\mathrm{y}$ una disminución del pico de masa ósea. Son elementos fundamentales en la prevención de la osteoporosis y de la osteopenia.

Ejercio físico.- La vida sedentaria aumenta la predisposición a la osteoporosis y osteopenia, este factor es otro punto importante en la prevención primaria.

\section{ESTRATEGIAS DE PREVENCIÓN}

La principal estrategia para afrontar la enfermedad y sus secuelas reside en la prevención, tanto primaria como secundaria. El planteamiento, en su mayor parte, irá orientado a generar hábitos de vida saludables, que nos ayuden a prevenir la patología.

Una dieta adecuada con aporte correcto de calcio y vitamina D disminuye el riesgo, y es una de las estrategias a seguir. El requerimiento nutricional adecuado de calcio es de 1000-1500 mg/ día, según la edad y momento del desarrollo.

Los niveles bajos prolongados de vitamina $\mathrm{D}$ producen una disminución de la absorción intestinal de calcio, e incrementan el recambio óseo. Las necesidades de vitamina D están cubiertas si la piel se 
expone a la luz solar o a radiación UVA, no precisando suplementos dietéticos ni farmacológicos. Como dato curioso las cremas fotoprotectoras impiden la síntesis de vitamina $\mathrm{D}$ activa.

Es recomendable corregir hábitos tóxicos, fundamentalmente, suprimir el tabaco y evitar el alcohol en grades cantidades. El consumo de alcohol moderado no tiene efectos tan claros sobre el hueso como el tabaco, pero está demostrado que dosis altas aumentan el riesgo de osteoporosis.

El ejercicio físico regular, especialmente el gravitatorio, es otra de las medidas primordiales para la prevención de la osteoporosis; ya que ayuda a recuperar parte de la masa ósea. El ejercicio físico se asocia con mayor masa ósea en niños y adolescentes.

Personas con índice de masa corporal baja (menor de $19 \mathrm{~kg} / \mathrm{m}^{2}$ ) tienen menos densidad mineral ósea, y por lo tanto, un alto riesgo de osteoporosis. Se debe evitar la delgadez extrema.

En mujeres, es especialmente importante tratar de evitar los periodos prolongados de amenorrea, ya que la disminución de la producción de estrógenos en estos periodos es muy acusada, y altera el recambio óseo a favor de la resorción ósea.

Si la causa de la osteoporosis es una patología médica o farmacológica, las medidas deben ir encaminadas al tratamiento y seguimiento de las mismas.

\section{BIBLIOGRAFÍA}

1.- Sanchez-Borrego R., Palacios S. "Prevención de fracturas osteoporóticas: La osteopenia como factor de riesgo". Ed. Habe Editores. S.L. Madrid 2005.

2.- Ferrandez L., Herrera A. "Fracturas osteoporóticas. Ed. Medical \& Marketing Communications". Madrid 2006.

3.- Rodríguez E. "Fracturas osteoporóticas". Ed. Panamericana. Madrid 2003.

4.- Quereda F., Mendoza N., Olalla M. A., Baró F., Durán M., " Enfoque profiláctico en mujeres posmenopáusicas asintomáticas: osteoporosis" MATURITAS. The European Menopaue Journal. Vol 52, Suplemento1, Noviembre 2005; (S42-S50).
Cuando existe una osteopenia y/o osteoporosis, se deben evitar actividades que puedan producir microtraumatismos óseos repetidos, y aquellas actividades que impliquen un riesgo de caídas, como la utilización de martillos neumáticos, la construcción el altura, evitar sobrecarga de peso, etc.... Todos estos datos deben tenerse en cuenta, especialmente desde el punto de vista de la prevención de riesgos laborales.

\section{CONCLUSIONES}

El dolor crónico de origen óseo tiene como consecuencia un acusado absentismo laboral, lo que se acompaña de un elevado gasto social y sanitario.

La osteoporosis y las fracturas osteoporóticas son hoy en día la "enfermedad silenciosa", debido a que siguen estando poco diagnosticadas y tratadas. Por ello debemos adoptar una actitud expectante e incluir programas de detección y prevención dentro del ámbito laboral.

Un correcto screening, diagnóstico y tratamiento de la osteoporosis conduce a una menor tasa de fracturas asociadas, pero aún así, es fundamental establecer programas efectivos de prevención, y estimularlos desde todos los ámbitos ; ya sea el personal, el laboral y el sanitario, para lograr su correcta optimización.
5.- O’Neill TW., Felsenberg D., Varlow J., Cooper C., Kanis J. A., Silman A. J. " The prevalence of vertebral deformity in European men and women: the European vertebral osteoporosis study. J Bone Miner Res 1996;11: 10101-8.

6.- Richy F., Ethgen O., Bruyere O., Mawt A., Reginster J Y. " Primary prevention of osteoporosis: mass screening scenario or pre-screening with questionnaires? An economic perspective. J Bone Miner Res. 2004; 19: 1955-60.

7.- Tosteson A, Solomon D, King AB, Dawson-Hughes B, Burge R, Wong J (2005) "Projections of osteoporosis fractures and costs by skeletal sites in the USA". ASBMR 27th annual meeting, Nashville, TN. 